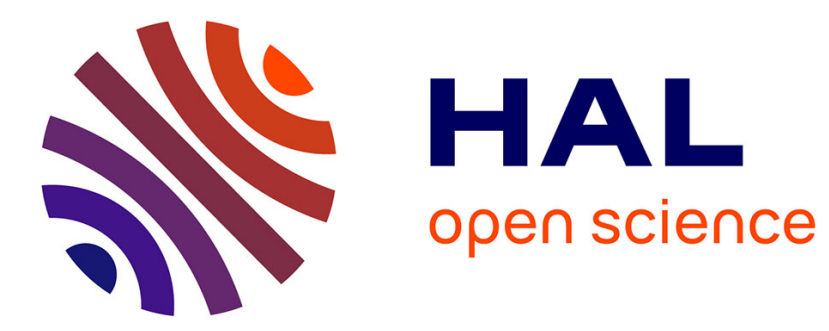

\title{
Les enjeux de la gravure pédagogique : préliminaires À l'étude du fonds d'estampes des frères Cacault (1803-1808)
}

\author{
Laëtitia Pierre
}

\section{- To cite this version:}

Laëtitia Pierre. Les enjeux de la gravure pédagogique : préliminaires À l'étude du fonds d'estampes des frères Cacault (1803-1808). La Collection Cacault, Publications de l'Institut national d'histoire de l'art, 2016, 10.4000/books.inha.6973 . halshs-03215874

\section{HAL Id: halshs-03215874 \\ https://shs.hal.science/halshs-03215874}

Submitted on 3 May 2021

HAL is a multi-disciplinary open access archive for the deposit and dissemination of scientific research documents, whether they are published or not. The documents may come from teaching and research institutions in France or abroad, or from public or private research centers.
L'archive ouverte pluridisciplinaire HAL, est destinée au dépôt et à la diffusion de documents scientifiques de niveau recherche, publiés ou non, émanant des établissements d'enseignement et de recherche français ou étrangers, des laboratoires publics ou privés. 
Les enjeux de la gravure pédagogique : préliminaires À l'étude du fonds d'estampes des frères Cacault (1803-1808)

\section{Laëtitia Pierre}

revues.org

Publisher

Institut national d'histoire de l'art

Electronic version

URL: http://inha.revues.org/6973

ISSN: 2108-6419

Electronic reference

Laëtitia Pierre, «Les enjeux de la gravure pédagogique : préliminaires À l'étude du fonds d'estampes des frères Cacault (1803-1808) », in Blandine Chavanne, Chantal Georgel and Hélène RousteauChambon (dir.), La Collection Cacault. Italie-Nantes, 1810-2010, Paris, INHA (" Actes de colloques ») 2016 [Online], Online since 15 February 2016, connection on 01 February 2017. URL : http:// inha.revues.org/6973

This text was automatically generated on 1 February 2017.

Tous droits réservés 


\title{
Les enjeux de la gravure pédagogique : préliminaires À l'étude du fonds d'estampes des frères Cacault (1803-1808)
}

\author{
Laëtitia Pierre
}

À partir du XVIII ${ }^{\mathrm{e}}$ siècle essentiellement, se développe un goût pour le dessin collectionné de plus en plus chèrement et diffusé de plus en plus largement grâce aux techniques de reproduction des modèles gravés ${ }^{1}$. Rassembler une collection de dessins d'artistes revient, depuis les années 1730 en Europe, à mettre en place un laboratoire dans lequel s'instaure une réflexion sur l'art et où il est dorénavant possible d'estimer l'œuvre et les spécificités artistiques des maîtres anciens. L'appropriation d'un savoir, voire d'une certaine capacité d'expertise et d'appréciation esthétique, peut dorénavant se mesurer au nombre et à la variété des représentations dessinées et reproduites en gravure, rassemblées dans une même collection. 
Fig. 1. Alexandre Roslin, Portrait de Dandré-Bardon, 1756, 83 x $67 \mathrm{~cm}$, Musée national des châteaux de Versailles et de Trianon, MV 5888.

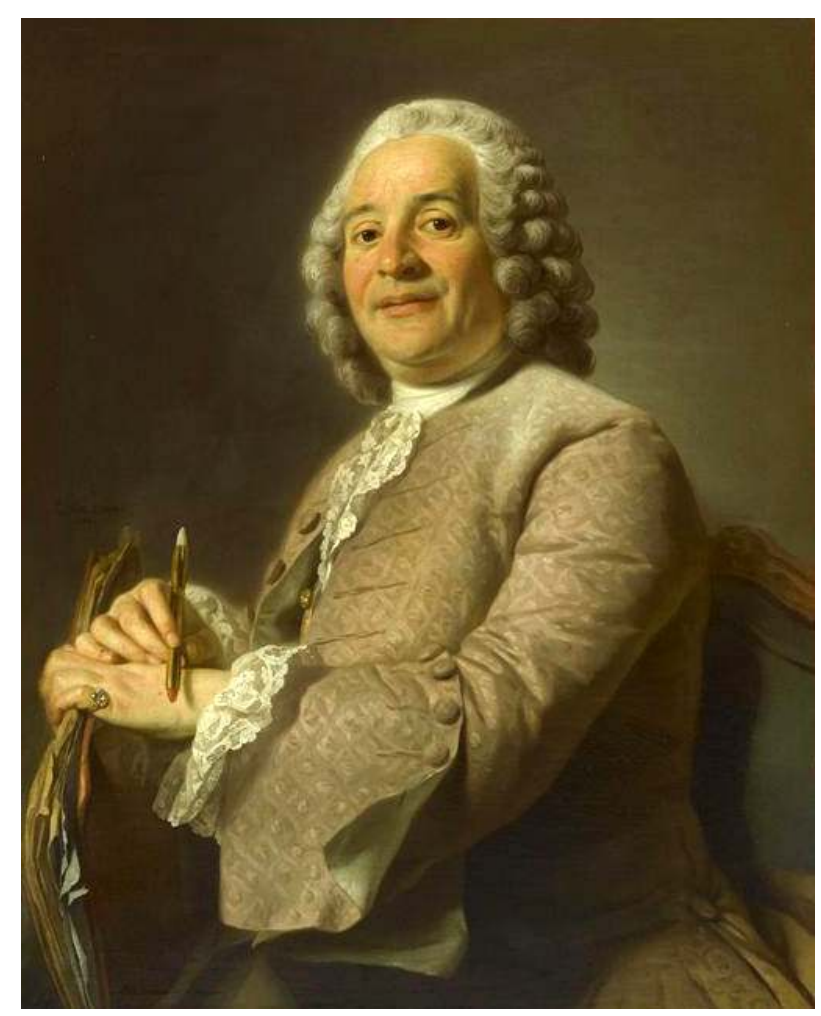

(c) RMN-Grand Palais (Château de Versailles) / Gérard Blot

2 Or, au lendemain de leur séjour en Italie, les frères Cacault composent et relient soixantequatre albums de gravures. Si cet amoncellement relève d'une démarche de collectionneur, l'entreprise d'édification du musée-école de Clisson prévoit une organisation qui privilégie la consultation d'un fonds d'estampes à visée documentaire dans le cadre de la formation des élèves ${ }^{2}$. Les sondages effectués par Maxime Préaud attestent de la qualité généralement moyenne des tirages ainsi que de leur état de conservation $^{3}$. Ces pièces se distinguent en revanche par leur nombre, la diversité des genres et des sujets gravés, voire la rareté de certaines, des qualités qui font d'elles un excellent moyen d'acquérir une connaissance substantielle de la production des écoles artistiques en Europe depuis la fin du Moyen Âge. En plus des ouvrages reproduisant les modèles gravés pour la copie d'après la figure humaine, la statuaire antique, les motifs géométriques, ornementaux, végétaux, etc., les porte-folios répertorient de nombreux sujets d'histoire et des paysages. De fait, nous proposons ici de mettre en rapport certains aspects constitutifs de ce fonds avec les modèles et les principes didactiques plébiscités notamment à l'occasion du programme de réforme de l'enseignement de l'Académie royale de peinture et de sculpture. La collection de gravures constituée par les frères Cacault entre 1803 et 1808 semble en effet relever des enjeux pédagogiques propres au siècle des Lumières à bien des égards si l'on se réfère à ses sources d'inspiration. Certains de ces principes mis en œuvre au sein de l'Académie de peinture ont pu constituer un modèle pour les frères Cacault, de même que certaines idées avancées par plusieurs artistes et enseignants de l'Académie, tel le peintre Michel-François Dandré-Bardon dont la réception pédagogique reste encore à estimer (fig. 1). 


\section{La recherche d'une nouvelle pédagogie}

3 Le 8 août 1793, lorsque David dénonce les méthodes d'enseignement de l'Académie royale comme des pratiques tortionnaires, il entérine un sentiment de défiance généralisée à l'égard de la pédagogie artistique ${ }^{4}$. David refuse de donner de nouvelles règles pédagogiques ${ }^{5}$ et abolit la stricte organisation des classes d'apprentissage du dessin auxquelles les écoles académiques devaient se référer au profit du seul atelier. L'inspiration philosophique de l'Émile de Rousseau et le postulat de la Nature, perçue comme réformatrice de la corruption des mœurs et de la pensée, est un substitut idéologique à la fois efficace et complaisant ${ }^{6}$.

Ce lien intime avec la Nature est repris explicitement par les Cacault. Les musées, liés organiquement et géographiquement aux écoles de formation, doivent être situés dans un site paysager privilégié et placées sous la direction d'un artiste confirmé, susceptible de conduire les cours pratiques de la copie d'après les modèles et d'après la nature ${ }^{7}$. D'ailleurs, le cadre naturel de Clisson est aménagé de manière à illustrer une vision à la fois bucolique et méditative de la nature et s'inscrit comme un véritable paradigme de la doctrine artistique promue par l'enseignement des Cacault. Cette conception, partagée par leurs contemporains à l'instar de Guillet de Saint Georges et Jacques-Louis David, cristallise à la fois la croyance en la revivification des puissances créatrices de la nature et entérine l'idéologie politique et philosophique révolutionnaire ${ }^{8}$. À y regarder de plus près, la revendication de ces conceptions ne tient pas tant au fait d'un renouvellement des savoirs et de la culture artistique qu'à une conception philosophique et esthétique. Cette quête de l'absolu par le biais des formes sensibles soulève un paradoxe lorsque se pose la question d'un renouvellement effectif des pratiques pédagogiques et de la transmission des modèles artistiques.

Comment le développement de la philosophie esthétique influence-t-il concrètement ces nouveaux concepteurs de programmes d'enseignement? Leur défiance vis-à-vis des débats critiques formulés au cours du XVIII ${ }^{\mathrm{e}}$ siècle les engagent-ils réellement à considérer différemment les sources utilisées en tant que modèles d'apprentissage ? Au-delà du rejet global de la production artistique conçue au cours des décennies précédentes, il n'est pas hasardeux d'affirmer que la plupart des revendications idéologiques et pédagogiques formulées par ces nouveaux directeurs sont le fruit des réformes et des expériences d'enseignement préalablement organisées en France grâce notamment aux écoles de dessin'.

En fait, dès 1747, sous l'administration de Lenormant de Tournehem, le comte de Caylus et le Premier peintre Charles-Antoine Coypel opèrent une refonte du corpus théorique et pédagogique au sein de l'Académie royale de peinture et de sculpture. À ces programmes s'ajoute la collaboration de littérateurs tels que l'abbé Batteux, Charles-Nicolas Cochin, Claude-Henri Watelet, et Michel-François Dandré-Bardon qui ménagent à partir du corpus de la littérature artistique (Roger de Piles, André Félibien, Henri Testelin, l'abbé du Bos, etc.), une réflexion sur la doctrine académique et l'enjeu de la pratique du dessin ${ }^{10}$ 


\section{La gravure au cœur de l'enseignement}

7 Le peintre et pédagogue Michel-François Dandré-Bardon consacre ainsi à partir de 1755 toute son énergie à cette entreprise et développe notamment des réflexions sur l'étude d'après les modèles gravés. Au fil de ses publications rédigées en 1756 et 1774, il valorise une grille de lecture méthodologique d'une œuvre complexe comme peut l'être une peinture d'histoire. Si son intention, à l'instar de celle des Cacault, n'est pas de remettre en question la valeur effective du principe de l'imitation de la Nature et de l'Antique, la formulation de sa doctrine ne s'inscrit pas uniquement dans un respect scrupuleux de la tradition platonicienne. Dandré-Bardon et les frères Cacault sont tous les trois nourris de l'influence d'une philosophie qui aborde depuis plusieurs décennies l'étude des systèmes de pensée et des modèles de représentation du monde sensible comme des réminiscences des mécanismes propres de la Nature. Nature et esprit de système ne sont pas antinomiques aux yeux de ces lecteurs avertis des ouvrages antinomiques de Condillac, Kant et Hegel; la représentation de l'univers sensible passe par l'établissement d'un savoir préalable sur lequel se fondent l'appréciation et l'invention des œuvres d'art ${ }^{11}$. Ainsi, le rassemblement considérable de gravures d'écoles, de périodes, de styles et de genres variés démontrent également l'intention de faire l'apologie d'une culture picturale et sculpturale savante, nécessaire à tous les praticiens, artisans, connaisseurs ou artistes. À l'évidence, l'imitation des maitres, au-delà de certaines objections d'ordre stylistiques ou formelles, est le second principe fondamental sur lequel repose le projet pédagogique des Cacault ${ }^{12}$.

8 En 1797, François Cacault affirme la nécessité de constituer un fonds documentaire de gravures et une collection de moulages, de pierres et de plâtres d'après l'Antique dans les écoles de province. Or, seuls les fonds gravés de l'Académie royale de peinture et de sculpture peuvent soutenir l'envergure de l'ambition pédagogique évoquée par Cacault. La constitution d'un tel fonds atteste l'influence caractéristique des discours sur la réforme de l'enseignement des Beaux-arts au sein de l'Académie royale de peinture et de sculpture. L'impulsion de ces programmes pédagogiques d'envergure, réalisés durant la seconde moitié du XVIII ${ }^{\mathrm{e}}$ siècle, a largement conforté l'idée de la nécessité de composer des fonds de gravures soigneusement médités dans les ateliers des professeurs. Ces fonds n'ont plus seulement une fonction de répertoire documentaire : ils permettent, tout en conservant un emploi pratique au sein des classes du dessin, d'apprécier un certain degré d'érudition et de répondre à la revendication du goût et du savoir-faire auxquels prétendent leurs concepteurs ${ }^{13}$. S'ils semblent sur certains points pédagogiques se fonder sur l'enseignement dispensé à l'Académie de peinture et de sculpture, les Cacault n'affirment pas moins de façon catégorique combien cet enseignement est obsolète : « Les vices de notre école des arts prennent leur origine dans l'institution de l'Académie de Paris, dans les premières leçons des maîtres, dans un système d'imitation et une routine pédantesque qui éloignent des idées simples de la nature qu'on perd de vue pour s'attacher à des règles, à une manière. Il faudra que tout rentre dans un ordre naturel ${ }^{14}$ ». 


\section{Les écoles de dessin, des modèles pédagogiques pour les Cacault}

Plus que l'enseignement académique, les Cacault semblent prendre pour base une autre expérience, celle développée au sein des écoles académiques de dessin répandues dans les grandes villes de France depuis le XviII ${ }^{\mathrm{e}}$ siècle essentiellement ${ }^{15}$. Dans ces écoles qui restent pour la plupart sous la férule de l'Académie et dispensent des enseignements destinés aux artisans, aux ingénieurs, voire à de futurs artistes, l'enjeu que revêt l'apprentissage des arts par le biais de la gravure est une préoccupation constante. Cependant, l'enseignement du dessin y variait sensiblement en fonction des intentions, des moyens et des conditions. Si elles prenaient préliminairement le soin de souscrire au modèle d'organisation instauré par l'Académie, ces classes n'avaient pas d'autre choix que de restreindre leur enseignement à un apprentissage technique. Ces principes étaient plus suivis dans les idées que dans les faits et variaient en fonction des besoins édictés par la ville qui attribuait généralement un financement à ces classes. La qualité de l'enseignement dispensé tenait également à la personnalité des directeurs, des professeurs et à leur degré d'investissement. Ils étaient libres d'insister sur des aspects plus spécifiques de la pratique du dessin, d'orienter en fonction de leurs propres ressources documentaires et de leurs intérêts artistiques l'étude de modèles particuliers, de valoriser le perfectionnement technique de certaines connaissances comme la géométrie, la perspective, parfois même les techniques de reproduction de la manière des maîtres. En restreignant cet enseignement à la pratique du dessin d'après le modèle inanimé, en conservant le droit d'édicter les règles d'organisation, de pratiques et de contrôle de la diffusion des modèles gravés sur lesquels reposent cet enseignement, l'Académie, institution tutélaire de ces écoles, se réservait l'exercice d'une autorité intellectuelle sur le contenu doctrinal des arts.

10 Le nombre de pièces de qualités et de dimensions variées du fonds Cacault ainsi que leur mode de classement nous placent sans conteste devant une entreprise pédagogique d'envergure. Mais le postulat de la gravure, choisie et méditée en tant que modèle et source de transmission, semble également dépassé par l'ambition philanthropique des Cacault. Leur projet d'organisation accorde au fonds la fonction d'un laboratoire visuel, véritable consortium de la connaissance des arts, de ses modèles et des diverses pratiques de transmission qui découlent de son étude. L'approche préliminaire peut d'ores et déjà mettre en perspective certains déterminismes conceptuels et méthodologiques, d'ordre à la fois culturel et politique. Ces principes suggèrent que les frères Cacault avaient préalablement étudiés les différentes pratiques de l'enseignement des Beaux-arts.

\section{Dandré-Bardon, un modèle pédagogique pour les Cacault ?}

L'étude de l'œuvre théorique et pédagogique de l'un des principaux acteurs de la réforme de l'enseignement officiel, Michel-François Dandré-Bardon, revêt un enjeu fondamental dans le cadre de l'exploration de ces problématiques. Lorsqu'en 1752 Dandré-Bardon est chargé de composer un système approfondi de la connaissance des Beaux-arts puis de dispenser son enseignement aux élèves de l'Académie et aux lauréats des Grands Prix, il 
est chargé de reformuler dialectiquement les mécanismes de création et d'invention de la peinture. S'il ne déroge en aucun cas aux principes de l'imitation savante de la Nature et des modèles classiques, il entreprend une démarche novatrice afin de structurer leur mise en pratique effective durant la période de formation des jeunes artistes, notamment lorsqu'ils emploient la gravure: pour lui, il s'agit de provoquer une stimulation mémorielle chez un artiste en train de composer une œuvre. Les travaux de DandréBardon, dans la pratique, ont joué un rôle majeur dans cette prise de conscience de la dialectique des mécanismes de la pensée et de la création artistique. Dandré-Bardon publie en 1765 un Traité de peinture suivi d'un essai sur la sculpture, pour servir d'introduction à une histoire universelle relative à ces Beaux-arts ${ }^{16}$. L'ouvrage inclut notamment une biographie des graveurs de l'école française qui suggère le plan de constitution d'un fonds documentaire et graphique. Dandré-Bardon précise alors que :

Quoique la gravure n'entre point dans le plan de cet ouvrage, ses principes sont si relatifs au dessin, à la composition, aux effets même du coloris ; ils contribuent tant à reproduire avec succès les chefs-d'œuvre des grands peintres et des sculpteurs fameux que nous avons cru qu'il convenait de faire mention des cultivateurs de cet art. Puisque par le talent de la pointe et du burin ils ont transmis jusqu'aux siècles les plus reculés les ouvrages des grands maitres, consacrons leurs noms à la postérité17.

Fig. 2. Jean Audran, La Hollande acceptant la Paix et se détachant de l'Allemagne, d'après Charles Le Brun, dessiné par Jean-Baptiste Massé, 1753, n³3, 39,5 x 79 cm, BNF, Estampes.

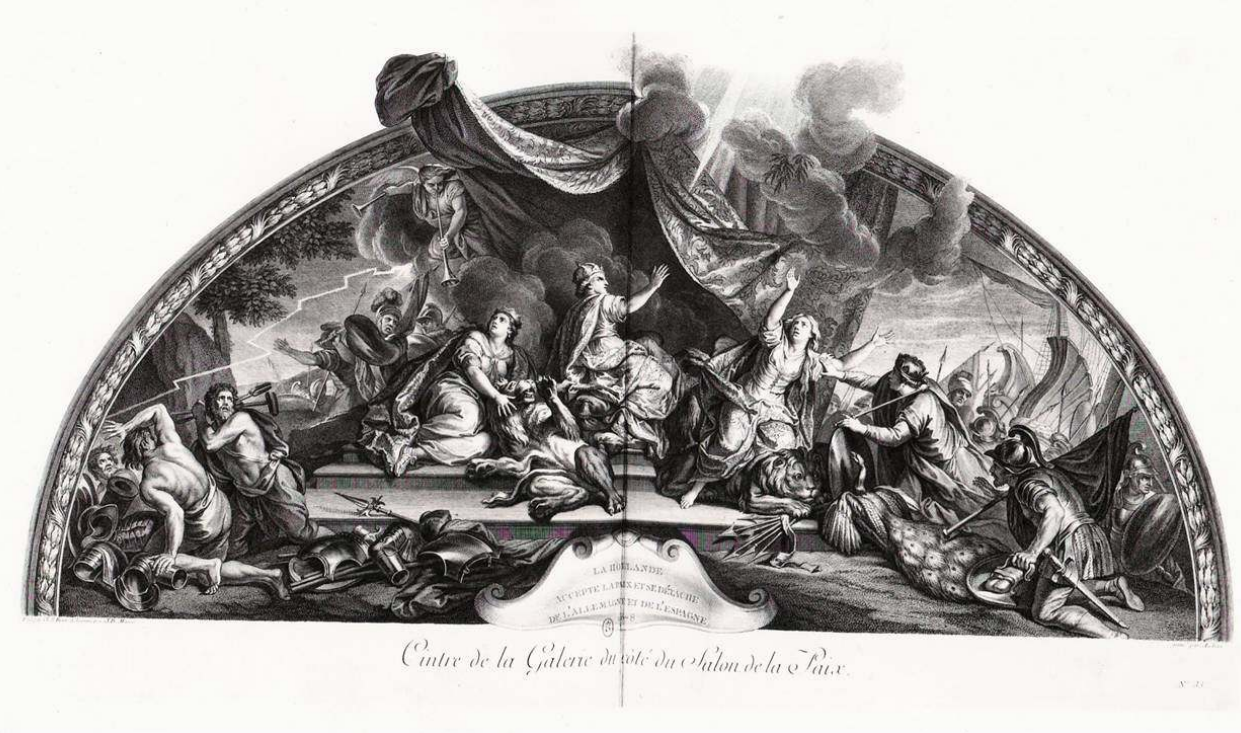


Fig. 3. Louis Roullet, Les saintes femmes au tombeau d'après Annibal Carrache, 1690, $41 \times 25,5$ cm, BnF, Estampes.

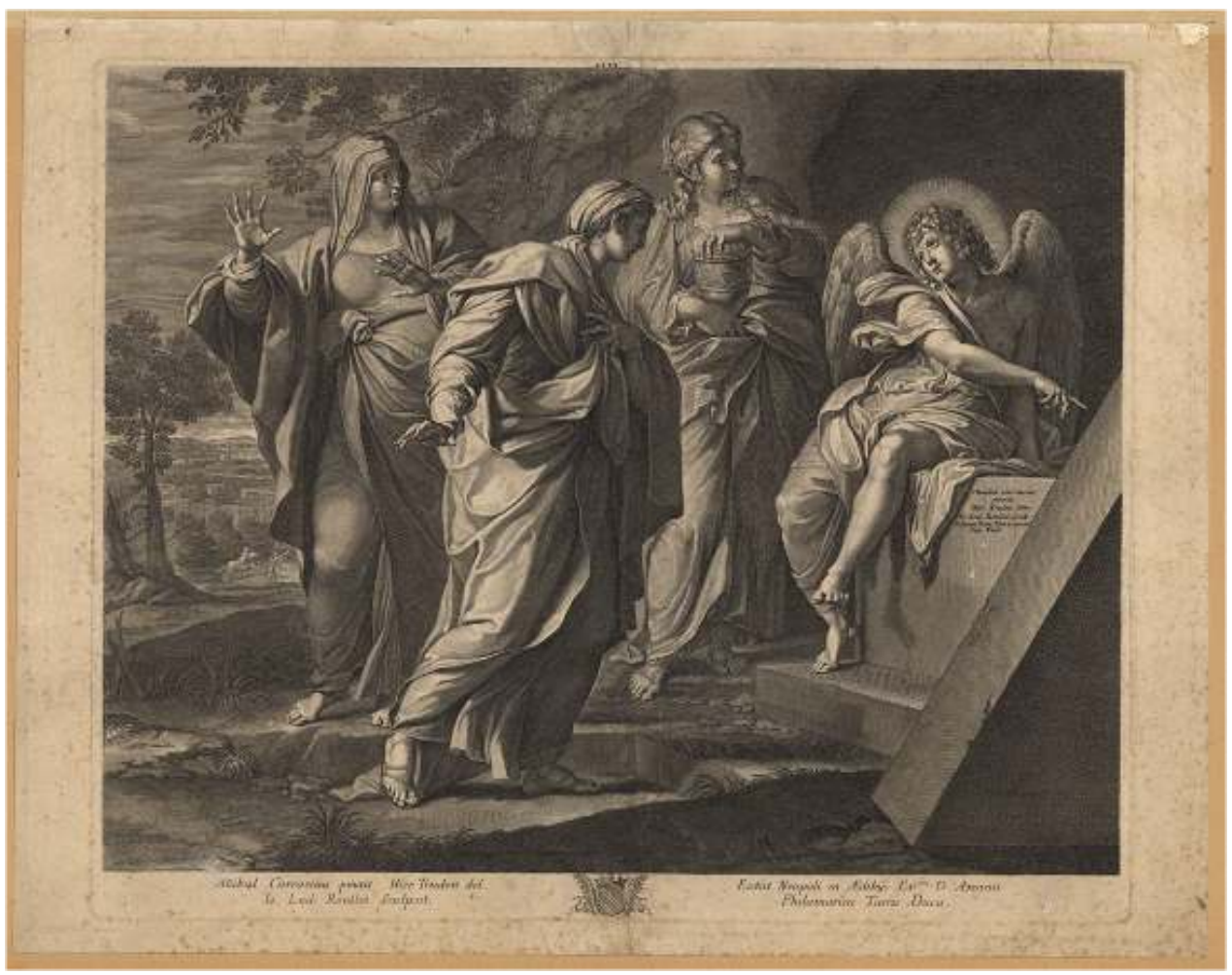

Photo BnF / Dép. Estampes

Dandré-Bardon procède à la sélection d'un nombre limité d'estampes grâce auxquelles les artistes peuvent désormais vérifier l'efficience des principes de l'art de peindre ${ }^{18}$. Parmi les productions des graveurs aptes à fournir des modèles de qualité pour l'exercice de la copie, il se fonde sur les travaux de Testelin pour son histoire de l'Académie, et sur ceux de son prédécesseur Lépicié qui avait lui-même rassemblé et lu des manuscrits sur les vies et les ouvrages des peintres de l'Académie. Dandré-Bardon rassemble tout d'abord un nombre important de compositions d'après les maitres italiens - Carrache, Poussin, Raphaël, Le Dominiquin, Pierre de Cortone, Guido Reni et Le Guerchin - puis de Français tels que Philippe de Champaigne, Le Sueur, Perrier, La Hyre, Le Brun, Mignard, Jouvenet et Watteau, sans omettre les œuvres de portraitistes de renom comme Rigaud et Largillière. Son étroite collaboration avec les graveurs Charles-Nicolas Cochin, Bernard Lépicié et Nicolas Tardieu lui permet de composer un répertoire référentiel et stylistique varié tout en restreignant le plus souvent à une référence la représentation d'artistes tels que François Lemoyne, Joseph Parrocel, Jean Restout, Nicolas Bertin ou Antoine Coypel. Dandré-Bardon prévient également son lectorat des défauts de certains graveurs réputés, tels Bernard Picart à qui il reproche le fini froid de ses œuvres de maturité, Jean Pesne ou encore Étienne Baudet dont il souligne le manque de noblesse dans le rendu des compositions. Cette démarche tente de susciter un esprit critique et d'établir les fondements pédagogiques préalables à l'acquisition d'un jugement de goût fondé sur des sources reconnues par les artistes et les amateurs. Le répertoire ne se limite pas à la gravure d'interprétation à visée documentaire. Dandré-Bardon choisit aussi certaines estampes originales telles que les Impostures innocentes du même Bernard Picart, plébiscitées par les connaisseurs, ou celles composées par Claude Mellan dont il cite 
l'exemple de la Sainte Face gravée d'un seul trait circulaire ${ }^{19}$. Outre ce répertoire des connaissances fondamentales des modèles de gravure, Dandré-Bardon n'oublie pas de recommander la consultation de plusieurs recueils tels que le Traité sur le dessin et la gravure d'Abraham Bosse, Les proportions de l'Antique de Gérard Audran, les divers morceaux de l'histoire naturelle gravés par Louis de Chatillon, le recueil de Jean-Baptiste Massé reproduisant La Grande Galerie de Versailles et les deux salons qui l'accompagnent, peints par Charles Le Brun ${ }^{20}$ (fig. 2). Il cite également un certain nombre de références parmi les estampes qui composent le recueil réédité en 1744 par P.-J. Mariette et gravé par J. Coelemans, d'après les œuvres de la collection du parlementaire aixois Jean-Baptiste II Boyer d'Eguilles ${ }^{21}$ ainsi que des compositions de graveurs provençaux à l'instar de Louis Roullet, natif d'Arles ; Dandré-Bardon semble à l'occasion puiser dans le répertoire de ses propres sources iconographiques lorsqu'il désigne plus particulièrement une composition d'après Annibal Carrache représentant les Trois Maries au tombeau dans laquelle il admire la vérité des sentiments (fig. 3).

13 Le pédagogue propose donc en 1765 un répertoire synthétique des modèles : il suggère un fonds de gravures d'interprétation approprié aux besoins de l'élève qui s'exerce à la copie. L'auteur établit de fait les bases d'une culture visuelle grâce à la connaissance de gravures également originales et largement plébiscitées par les connaisseurs. La stricte limitation du nombre de modèles cités dans ce corpus suggère à l'artiste, désormais familiarisé avec les différentes techniques de gravure et les qualités et défauts respectifs des graveurs, de compléter ce fonds dans les limites du caractère normatif du jugement de goût.

14 Face à une critique de plus en plus virulente, Dandré-Bardon veut en fait démontrer que les élèves disposent désormais des connaissances et des moyens d'évaluer les modèles artistiques étudiés durant leur formation. Cette revendication ferme précède l'annonce d'un troisième et dernier volume entièrement consacré à l'étude du costume. En effet, s'il considère l'intérêt spécifique de la gravure en fonction de chacune des étapes de la formation de l'élève, Dandré-Bardon souhaite avant tout fournir aux artistes les ressources intellectuelles leur permettant de constituer des fonds documentaires spécifiques au projet de carrière qu'ils doivent désormais méditer dès les premiers mois de leur apprentissage. Il rejoint en ce sens Jacques-Nicolas Tardieu qui, le 3 juillet 1756, dans son discours Sur les dispositions nécessaires aux élèves ${ }^{22}$, rappelle combien il est fondamental d'éveiller chez l'élève une conscience critique vis-à-vis de ce qu'il copie, audelà de l'acquisition des principes nécessaires à la copie des modèles dessinés et gravés d'après les maîtres. Il recommande notamment aux élèves d'entretenir cette vivacité intellectuelle par l'étude littéraire propre à stimuler le jugement critique. Ceci implique également de la part du professeur qui dirige la classe un échange oral régulier sur des préceptes qui doivent accompagner l'exercice ${ }^{23}$. Tardieu insiste sur l'importance de l'éveil intellectuel du jeune copiste. Son avis précède ainsi de deux mois la présentation du programme des cours d'histoire, de géographie et de la fable dispensé par Dandré-Bardon qui décrit l'organisation et le déroulement pédagogique de la classe du dessin et du modèle en résumant les recommandations de son collègue :

C'est d'après les desseins d'habiles artistes qu'un jeune homme doit se former dans le maniement du crayon, et lorsque, ayant pris une bonne façon de dessiner, il se sera fortifié par l'étude d'après le modèle, alors il sera nécessaire qu'il étudie soit en gravant soit en dessinant au crayon ou à la plume, d'après des ouvrages gravés de bon goût, pour se former une manière de rendre avec le burin et la pointe la beauté d'un dessein ou d'un tableau ${ }^{24}$. 

pédagogique que peuvent mettre en avant les frères Cacault dans leur musée-école de Clisson, compte-tenu de la variété des gravures conservées...

Lorsqu'en 1760, les littérateurs composent un véritable répertoire de la pédagogie artistique sur le modèle élaboré par Dandré-Bardon, ils proposent d'établir les règles didactiques d'un enseignement mais aussi les modes de transmission dialectique du savoir-faire des grands maitres. Porte-paroles et garants de l'autorité de l'Académie royale de peinture et de sculpture, ces ouvrages doivent permettre à un jeune artiste d'atteindre le plus haut degré de maîtrise de la production picturale et sculpturale. En élaborant une propédeutique des modalités de transfert du savoir des maîtres aux élèves, cette littérature favorise aussi les conditions du renversement de l'autorité académique vers les écoles et les ateliers d'artistes dès 1793.

En adoptant ce modèle pédagogique, des réformateurs de l'enseignement académique comme les frères Cacault s'accaparent les normes de la culture et du jugement esthétique édictées sur les fondations de la pensée humaniste. La revendication et la constitution de vastes collections de gravures d'interprétation à l'instar de celle du musée-école de Clisson illustrent cette problématique du transfert de la légitimité artistique selon les normes prescrites d'après l'observation de la Nature ${ }^{25}$. Ces différents niveaux de lecture et leur mise en relation avec les réflexions théoriques d'un artiste tel que Dandré-Bardon renvoient à un faisceau de comparaisons historiques, littéraires, poétiques et artistiques qui interpellent aussi bien l'artiste en amont du processus d'invention et d'exécution d'une œuvre que l'amateur et le connaisseur ; l'idéologie et le projet d'école des frères Cacault furent sans doute également nourris par les vastes ramifications de cette culture artistique. L'exemple des gravures étudiées et composées par Dandré-Bardon ouvre un champ de questionnements sur une autre pratique de la gravure, celle qui consiste à ne plus étudier l'œuvre dans la logique de la stricte analogie formelle mais autorise une possible remise en question des stratégies de classifications stylistiques fondées sur la distinction entre des modèles qui soutiendraient l'émulation et l'imagination, et d'autres qui interviendraient plus spécifiquement dans le cadre d'un apprentissage technique. Il ne serait dès lors plus nécessaire de prendre la défense de ce type d'ouvrage contre les revendications d'auteurs concurrents qui espéraient une uniformisation stylistique des répertoires de gravures, notamment des figures antiques. Cette dimension spéculative que les Beaux-arts apportent à la fin du XVIII ${ }^{\mathrm{e}}$ siècle au développement des sciences historiques a selon toute vraisemblance inspiré plusieurs générations de connaisseurs philanthropes et d'élèves tout au long du siècle suivant ${ }^{26}$.

\section{NOTES}

1. Voir notamment les travaux de Marianne Roland Michel, Le dessin français au XVIII ${ }^{e}$ siècle, Fribourg, 1987, p. 243-253 ; Gady, B., «Gravure d'interprétation et échanges artistiques. Les estampes française d'après les peintres italiens contemporains (1655-1724) », Studiolo, vol. 1, 2002, p. 64-104) en 1679; Michel, C., "Le goût pour le dessin en France aux XVII ${ }^{\mathrm{e}}$ et XVIII ${ }^{\mathrm{e}}$ siècles : de 
l'utilisation à l'étude désintéressée », Revue de l'art, n¹43, 2004, p. 27-34 ; Christian Michel, «Les débats sur la notion de graveur/traducteur en France au XVIII siècle », Delineavit et Sculpsit. Dixneuf contributions sur les rapports dessin-gravure du XVI ${ }^{e}$ au XXe siècle, Mélanges offerts à Marie-Félicie Perez-Pivot,Lyon, PUL, 2003, p. 151-161.

2. Gaillard, C., Les estampes françaises de la collection Cacault du musée des Beaux-arts de Nantes. Reflets du goût d'un collectionneur à la fin $d u X_{\text {XVIII }}^{e}$ siècle, mémoire de maitrise d'histoire de l'art, Christine Prigent (dir.), université Paris-I-Panthéon-Sorbonne, 1996 ; Bonnard, I., Coural, N., Gérin-Pierre, C., «La restauration des albums Cacault du musée des Beaux-arts de Nantes », Technè, n²1, 2005, p. 14-18 ; Brégeon-Henwood, F., Les gravures d'après Jean-Baptiste Greuze dans la collection Cacault, mémoire de Master 1, Hélène Rousteau-Chambon (dir.), Université de Nantes, 2009. Nous remercions ici Hélène Rousteau-Chambon pour son aide précieuse durant la préparation de nos travaux et de nos réflexions ainsi que Flore Brégeon-Henwood qui nous a communiqué le résultat de ses sondages du fonds Cacault.

3. Voir le texte de Maxime Préaud dans le présent volume.

4. David, J.-L., « Sur la nécessité de supprimer les Académies », 8 août 1793, Lichtenstein, J. (dir.) La peinture, Paris, Larousse, 1995, [1993], p. 769-771.

5. Voir notamment Starobinski, J., Jean-Jacques Rousseau: la transparence et l'obstacle, Paris, Gallimard, 1971, p. 44-48.

6. Ibid., p. 44-48.

7. Sarrazin, B., Catalogue raisonné des peintures italiennes du musée des Beaux-arts de Nantes XIII - XVIII siècles, Paris, Réunion des musées nationaux, 1994, p. 60 et 65 ; AMAE, CP Rome, vol. 923, fol. 8.

8. Voir notamment Monnier, G., L'art et ses institutions en France. De la Révolution à nos jours, Paris, Gallimard, 1995 ; Starobinski, J., 1789. Les emblèmes de la raison, Paris, Skira, 1973, p. 108-121; Ehrard, J., L'idée de nature en France dans la première moitié du XVIII siècle, Paris, Albin Michel, 1994 [1963] ; Annie Becq, Genèse de l'esthétique française moderne. De la raison classique à l'imagination créatrice. 1680-1814, Paris, Albin Michel, 1994 [1984].

9. Nous renvoyons notamment aux récents travaux d'Aude Henry-Gobet consacrés à l'école académique de Rouen : Une sociabilité du dessin au XVIII siècle. Artistes et académiciens à Rouen au temps de Jean-Baptiste Descamps, 1715-1791, thèse de doctorat, Rabreau, D. (dir.), Paris 1-PanthéonSorbonne, 2007.

10. Voir notamment Michel C.et Germer, S. (dir.), « La naissance de la théorie de l'art en France », Revue d'esthétique, $\mathrm{n}^{\circ} 31 / 32,1997$; Griener P. et Hurley, C., « Une norme en transformation. La systématique du vocabulaire artistique au XVIII ${ }^{\mathrm{e}}$ siècle ", L'art et les normes sociales au XVIII ${ }^{e}$ siècle, Paris, éditions de la Maison des Sciences de l'homme, Passages//Passagen, 2001, vol. 2, p. 3-14.

11. Nous renvoyons ici notamment aux analyses de Michel Foucault à propos du système de représentation du signe aux $\mathrm{XVII}^{\mathrm{e}}$ et $\mathrm{XVIII}^{\mathrm{e}}$ siècles dans Les mots et les choses. Une archéologie des sciences humaines, Paris, Gallimard, 2010 [1966], p. 72-77.

12. Sur la question de l'imitation des maîtres d'après la gravure, nous renvoyons aux travaux de Henry, C., Aux sources du style. L'imitation et le culte des grands maîtres dans la peinture française de 1708 à 1799, thèse de doctorat, Rabreau, D. (dir.), université Paris 1-Panthéon-Sorbonne, 2002.

13. Voir notamment : Feix, F., « Le rôle de l'estampe dans l'enseignement de l'Académie royale de peinture. Toulouse et le Midi toulousain entre ciel et terre du Moyen Âge à nos jours ", (actes du 47e congrès de Toulouse : les 11, 12 et 13 juin 1993) ; Dendoven, D., Les collections d'artistes à Bruges au XVIII ${ }^{e}$ siècle : miroirs d'un goût changeant et matériaux pédagogiques? Collectionner dans les Flandres et la France du Nord au XVIII siècle, Lille (FRA), Université Charles-de-Gaulle-Lille 3, 2005 ; Jobert, B., «Collections et collectionneurs d'estampes en France de 1780 à 1880 d'après les catalogues de vente ", Collections et marché de l'art en France 1789 - 1848, Rennes, P.U.R., 2005, p. 243-255 ; Garcia, A.-M., « De l'art de graver. Prix de Rome et envois de gravure. ", L'éducation artistique en France. Du modèle académique et scolaire aux pratiques actuelles XVIII ${ }^{e}$ XIX ${ }^{e}$ siècles, Rennes, P.U.R., 2010, p. 37-52 
14. Sarrazin, B., op. cit., p. 51 et 64 .

15. Nous renvoyons notamment le lecteur aux ouvrages de Jean Locquin, La peinture d'histoire en France de 1747 à 1785. Étude sur l'histoire de l'évolution des idées artistiques dans la seconde moitié du XVIII ${ }^{e}$ siècle, Paris, 1978 [1912] ; Pevsner, N., Academies of art. Past and present, Cambridge, 1973 [1940] ; Roche, D., Le siècle des Lumières en province. Académies et académiciens provinciaux, 1680-1789, 2 vols., Paris et La Haye, 1978 ; Children of Mercury. The education of artists in the Sixteenth ans Seventeenth centuries, Providence, Department of Art, Bell Gallery, List Art Center,Brown University (March 2 - March 30 1984), 1984 ; Bignamini, I., «Jean-Bernard Le Blanc et l'Académie anglaise de 1749 ", Revue de l'art, 1986, n73, p. 17-27 ;Heinich, N., Du peintre à l'artiste, artisans et académiciens à l'âge classique, Paris, 1993 ; Lahalle, A., Les écoles de dessin au XVIII ${ }^{e}$ siècle, Rennes, PUR, 2006, p. 227-231. Il faudrait aussi ajouter Michel, C., L'académie royale de peinture et de sculpture (1648-1793), La naissance de l'École française, Genève, Droz, 2012.

16. Dandré-Bardon, M.-F., Traité de peinture suivi d'un essai sur la sculpture, pour servir d'introduction $\grave{a}$ une histoire universelle relative à ces Beaux-arts, Paris, Saillant libraire, 1765, $8^{\circ}, 2$ vols.

17. Ibid., p. 200-201.

18. Ibid., «Catalogue des plus fameux peintres, sculpteurs et graveurs de l'école française morts jusqu'en 1765 », Section III. p. 200-227.

19. Henry, C., « La leçon posthume de Bernard Picart », op. cit., 2002, p. 175-192.

20. La Grande Galerie de Versailles et les deux salons qui l'accompagnent, peints par Charles Le Brun, (...) dessinés par Jean-Baptiste Massé, (...) et gravés sous ses yeux par les meilleurs maîtres du temps, Paris, 1753.

21. Recueil d'estampes d'après les peintres les plus célèbres d'Italie, des Pays-Bas et de France qui sont à Aix dans le cabinet de M. Boyer d'Aguilles, gravées par Jacques Coelemans d'Anvers, avec une description de chaque tableau et le caractère de chaque peintre, Paris, chez P.-J. Mariette, 1744 區1709医.

22. Tardieu, J.-N., Sur les dispositions nécessaires aux élèves, discours lu devant l'Académie royale de peinture et de sculpture le 3 juillet 1756, ENSBA, mss 199.

23. ENSBA, mss 199 «L'usage unique que les élèves devraient faire des estampes bien exécutées d'après de grands maîtres serait d'y étudier la bonne manière de composer, les effets de lumière et la correction du dessein, sans cependant devenir plagiaire en copiant des groupes ou des compositions entières ; mais il s'agit ici des élèves qui n'ont point assez étudié pour imiter le bon goût d'un ouvrage sans en prendre la composition. Je dis donc pour revenir à mon sujet, qu'une estampe artistement gravée d'après un grand maître donnera aux peintres et aux sculpteurs la composition, la distribution des lumières, les effets piquants, une touche de dessein étudiée et hardie ".

24. Ibid.

25. On voit notamment ces débats s'articuler autour de la refonte des grands prix au lendemain de la Révolution et conditionner aussi bien le statut de l'élève-graveur que celui du peintre de grand genre, reconnus et appréciés par la production de peintures et de scènes de paysage. Voir à ce sujet : Stefani, C., «Études d'après nature et tableaux composés. Les débats à l'Académie des Beaux-arts, dès l'institution à la suppression du Grand prix de peinture de paysage historique (1817-1863) ", Studi di Storia dell'arte, 2000, n¹1, p. 221-240.

26. L'ouvrage d'André Lens, Le costume ou essai sur les habillements et les usages de plusieurs peuples de l'antiquité prouvé par les monuments - par André Lens, peintre - Liège, J.-F. Bassompierre, 1776, est rédigé au moment où Dandré-Bardon commence à publier les premiers cahiers de son Costume. Lens justifie la publication de ses travaux par la réforme qu'ils proposent des connaissances transmises par les ouvrages sur les antiquités, principalement ceux du comte de Caylus et de Winckelmann. Sous couvert d'une justification d'ordre scientifique, Lens défend un postulat stylistique opposé à celui de Dandré-Bardon, fondé sur une revendication explicite du savoir de l'antiquaire. 
AUTHOR

LAËTITIA PIERRE 